\title{
Optimal Control for a Stationary Population
}

\author{
Ming $\mathrm{Li}^{1} \&$ Qing Xie ${ }^{1}$ \\ ${ }^{1}$ Basic Courses Teaching Department, Anhui Wenda Information Engineering Institute, Hefei, China \\ Correspondence: Ming Li, Basic Courses Teaching Department, Anhui Wenda Information Engineering Institute, \\ Hefei, China. Tel: 152-5695-8571. E-mail: liming5165@126.com
}

Received: April 6, 2012 Accepted: May 15, 2012 Online Published: July 26, 2012

doi:10.5539/jmr.v4n4p140 URL: http://dx.doi.org/10.5539/jmr.v4n4p140

\begin{abstract}
As one of the most important achievements in nonlinear science, population system has drawn wide attention and extensive research in the past few decades. However, population control is a systematic social project with much complexity for the reason that it involves knowledge in many aspects, such as functional analysis, differential equations, partial differential equations, operator theory. Through initiating a series of groundbreaking work on the issue of population control in China, our scientific workers have made a lot of achievements, which are valuable in terms of theory and practice, in understanding and addressing this issue in a correct way.

To conduct intensive research on the issue of optimal control is a right way to achieve that. They have made strict and detailed analysis to population system, whose results have a great influence on the family planning policy in China.

This paper starts from deducing the population equation and explaining its parameters meaning. Next, it gives the answer to a simple model. Based on stationary population model, this paper, considering population mortality and gaining factor (can only depend on age), gives its prediction to a more general case and tries to gain the optimal control towards population.
\end{abstract}

keywords: stationary population, optimal control, gaining factor

\section{Introduction}

Overpopulation has always been the issue that Chinese government lays much emphasis on. It is also a serious crisis we are facing at present. The problems in basic necessity of life and employment as well as the shortage of natural resources brought by overpopulation have become great constraints in the development of Chinese economy. What's worse, it has brought society many problems as the overpopulation of rural labor forces have already caused. Since the policy of family planning was carried out in the 1970s, better control over the population has been achieved and the rate of population increase has decreased intensively. This will be more persuasive if it is analyzed from the perspective of statistics. It is necessary to analyze the population issue in the angle of mathematics, and that is, predicting and controlling the population by gaining population index from the number of population.

At the end of the 1970s and the beginning of the 1980s, Song Jian and other people began to analyze population issue from the perspective of mathematics. They established the partial differential equation model in the development of population, analyzed parameter equation like the mortality function and fertility function parameter equation, and answered the numerical value on the basis of population development equation. By doing so, it can be used to predict the number of population and various population indexes, such as birth rate, mortality, index of aging, number of the aged, number of labor force, index of labor force. All of these will bring Chinese population research into quantitative analysis and research from the pure qualitative analysis.

The optimal control on population system with age structure has been researched extensively in recent decades as many domestic and foreign scholars have done much work in this field. To our knowledge, many workers have devoted themselves in researching the optimal control since M. Brokata researched the optimal control with age structure and population dynamic system for the first time. Population system has formed into a huge system, spreading itself into the vast sea of documents.

The paper explains the optimal control of the dense population.Especially for the stationary population, this paper explains the meaning of the parameters and I obtain the optimal control by the theory of partial differential 
equations. I do some detailed study of a population equation, which is established on the emergence of the birth rate, mortality rates, gain factor (not depended on age) and other parameters. I prove the existence of the optimal control by the control convergence theorem.

The optimal control on population system with age structure has been researched extensively in recent decades as many domestic and foreign scholars have done much work in this field. To our knowledge, many workers have devoted themselves in researching the optimal control since M. Brokata researched the optimal control with age structure and population dynamic system for the first time. Population system has formed into a huge system, spreading itself into the vast sea of documents.

The paper explains the optimal control of the dense population. Especially for the stationary population, this paper explains the meaning of the parameters and I obtain the optimal control by the theory of partial differential equations. I do some detailed study of a population equation, which is established on the emergence of the birth rate, mortality rates, gain factor (not depended on age) and other parameters. I prove the existence of the optimal control by the control convergence theorem.

\section{Derivation of Population Equation}

The population issue is one that many people show their interest to. The ordinary differential equation model has often been used in the research of population development. The model only takes the total number of the population and its aggregate growth rate into consideration, and the age structure of population is not included. As a matter of fact, the age structure of population has a great effect on the change of the number of population. If you compare the youth-oriented society and the old age-oriented society, you will find great differences in the changing of population. In order to take the age structure into account, it is necessary to approach partial differential equation model. Here is the continuous model which considers the age of population.

Let $x, t$ said the age, time, and function $N(x, t)$ expressed the total number of population in the age of less than $x$ at $t$. And let function $N(x, t)$ on $x, t$ is continuously differentiable, in which $a_{m}$ is the maximum age, $N(t)$ is the total number of population at $t$. Obviously, $N(x, t)$ has the following properties:

(1) $N(0, t)=0$;

(2) To any a $b \geq a_{m}, N(b, t)=N(t)$.

Then let $p(x, t)=\frac{\partial N(x, t)}{\partial x}$, call $p(x, t)$ as the population density function. Due to function $N(x, t)$ about $x$ is not reduced and the above-mentioned properties (1) and (2), population density function $p(x, t)$ has the following properties:

$(1)^{\prime} p(x, t) \geq 0$;

$(2)^{\prime}$ When $b \geq a_{m}, p(b, t)=0$.

When the population density function $p(x, t)$ is known, apparently,

$$
N(x, t)=\int_{0}^{x} p(y, t) d y, N(t)=\int_{0}^{a_{m}} p(y, t) d y
$$

and the number of population is $\int_{x_{1}}^{x_{2}} p(y, t) d y$ when the age is in the $\left[x_{1}, x_{2}\right]$.

The mortality is different for people in different age, thus mortality function has a great influence on the population development. Regard $M(x, \Delta x, t)$ is the average death toll of those whose age is between $[x, x+\Delta x]$ at the unit time $t$, and the mortality function can be defined as:

$$
\mu(x, t)=\lim _{\Delta x \rightarrow 0} \frac{M(x, \Delta x, t)}{\int_{x}^{x+\Delta x} p(y, t) d y}
$$

Set $p(x, t)$ as continuous and differentiable function. When $\Delta x, \Delta t$ is small to the full, in the $t$ to $t+\Delta t$ time, the number of death of the people whose age are between the $x$ and the $x+\Delta x$ is

$$
\mu(x, t) p(x, t) \Delta x \Delta t,
$$

And the age of the survived are between $x+\Delta t$ and $x+\Delta x+\Delta t$ at $t+\Delta t$. Thus it can be deduced that

$$
p(x, t) \Delta x-p(x+\Delta t, t+\Delta t) \Delta x=\mu(x, t) p(x, t) \Delta x \Delta t+\alpha(\Delta x, \Delta t),
$$


In which, the $\alpha(\Delta x, \Delta t)$ is infinitesimal quantity at $\Delta x \rightarrow 0, \Delta t \rightarrow 0$. The above function can be rewritten into:

$$
p(x+\Delta t, t+\Delta t) \Delta x-p(x, t+\Delta t) \Delta x+p(x, t+\Delta t) \Delta x-p(x, t) \Delta x=-\mu(x, t) p(x, t) \Delta x \Delta t+\alpha(\Delta x, \Delta t),
$$

Both sides are divided by $\Delta x \Delta t$ at the same time and make $\Delta t \rightarrow 0$, and the following can be expressed like this:

$$
\lim _{\Delta t \rightarrow 0} \frac{p(x+\Delta t, t+\Delta t)-p(x, t+\Delta t)}{\Delta t}=\frac{\partial p(x, t)}{\partial x},
$$

Thus

$$
\frac{\partial p(x, t)}{\partial x}+\frac{\partial p(x, t)}{\partial t}=-\mu(x, t) p(x, t)
$$

Equation (1) is partial differential equations of first order. In order to work out the population density function $p(x, t)$, initial conditions and boundary conditions also should be given. Set the latest census time is $t=0$, and $p(x, 0)=p_{0}(x)$ is known. Set $p_{0}(t)=\varphi(t)$ is the birth population at unit time $t$, and when $\varphi(t)$ is known, the mathematical model for population is

$$
\left\{\begin{aligned}
\frac{\partial p(x, t)}{\partial x} & +\frac{\partial p(x, t)}{\partial t}=-\mu(x, t) p(x, t) \\
p(x, 0) & =p_{0}(x), p(0, t)=\varphi(t) \\
p\left(a_{m}, t\right) & =0 .
\end{aligned}\right.
$$

The Equation (2) can be addressed as the continuous equation on population development.

\section{The Solution of the Model}

When predicting the future population situation in short term, we often address it in a simple way: assuming that the social conditions remains relatively stable over a period of time, the mortality function $\mu(x, t)=\mu(x)$ has nothing to do with time and it is only a function of one variable regarding age as its variable quantity. The death function of the following years can be replaced by the mortality function of the starting year. This simple operation makes it easy to work out population development equation, which helps to predict population in short term in a highly accurate way.

A relatively stable society refers to a society remains unchanged in sex ratio of population, childbearing patterns of women, birth rate and mortality for a long time when social conditions remained relatively stable.

Here, set $b(x)$ is birth rate, and it is only associated with age and has nothing to do with time $t$. Mark $a(>0)$ is the minimum age of child-bearing, then

$$
p(0, t)=\int_{a}^{a_{m}} b(y) p(y, t) d y
$$

This is a boundary condition and the Equation (2) becomes

$$
\left\{\begin{array}{l}
\frac{\partial p(x, t)}{\partial x}+\frac{\partial p(x, t)}{\partial t}=-\mu(x, t) p(x, t) ; \\
p(x, 0)=p_{0}(x), p(0, t)=\int_{a}^{a_{m}} b(y) p(y, t) d y \\
p\left(a_{m}, t\right)=0 .
\end{array}\right.
$$

And it also assumes that the society is closed, and that is to say, the migration rate is $f(a, t)=0$.

Here suppose the $p_{0}(x)$ meets the following consistency condition

$$
p_{0}(0)=\int_{a}^{a_{m}} b(y) p_{0}(y) d y
$$

And the following equation is acceptable

$$
p_{0}\left(a_{m}\right)=0 \text {. }
$$

To get the solution easily, first make a substitution of unknown function, and the equation is homogeneous. Make

$$
q(x, t)=p(x, t) e^{\int_{0}^{x} \mu(\tau) d \tau},\left(t \geq 0,0 \leq x \leq a_{m}\right)
$$


Then Equation (3) can be evolved into

$$
\left\{\begin{array}{l}
\frac{\partial q(x, t)}{\partial x}+\frac{\partial q(x, t)}{\partial t}=0, t>0,0<x<a_{m} ; \\
q(x, 0)=q_{0}(x)=p_{0}(x) e^{\int_{0}^{x} \mu(\tau) d \tau}, 0 \leq x \leq a_{m} ; \\
q(0, t)=\int_{a}^{a_{m}} B(y) q(y, t) d y, t \geq 0 .
\end{array}\right.
$$

In which,

$$
B(x)=b(x) e^{-\int_{0}^{x} \mu(\tau) d \tau}
$$

and compatible condition (4) can be turned into Here we use method of characteristic curves in solving Equation (6), and the characteristic equation is

$$
\frac{d \chi(t)}{d t}=1
$$

So the characteristic curve is $\chi(t)=t+x_{0}-t_{0}$ crossing the point $\left(x_{0}, t_{0}\right)$, and $q(x, t)$ along characteristic lines differential is

$$
\frac{d q(\chi(t), t)}{d t}=\frac{\partial q}{\partial t}+\frac{\partial q}{\partial x} \frac{d \chi(t)}{d t}=\frac{\partial q}{\partial t}+\frac{\partial q}{\partial x}=0 .
$$

Namely it is constant along any feature line. The following is the discussion of the expression of solution in different regions.

In the region $\left\{(x, t) ; t \leq x<a_{m}, t \geq 0\right\}$, the feature line crossing any point $(x, t)$ must intersect the initial interval $0 \leq x<a_{m}$ in the decreasing direction of $t$. So it is

$$
q(x, t)=q_{0}(x-t),\left(t \leq x<a_{m}, t \geq 0\right) .
$$

In the region $\left\{(x, t) ; t-a \leq x \leq t, t \geq 0,0 \leq x<a_{m}\right\}$, the characteristic lines crossing any point $(x, t)$ must intersect the point $t-x$ on the zone $[0, a]$ in $t$ axis in the boundary region in the decreasing direction of $t$. Hence, from boundary condition

$$
\begin{aligned}
& q(x, t)=q(0, t-x) \\
& =\int_{a}^{a_{m}} B(y) q(y, t-x) d y \\
& =\int_{a}^{a_{m}} B(y) q_{0}(y-t+x) d y .
\end{aligned}
$$

This is because of $0 \leq t-x \leq a, a \leq y \leq a_{m}$, and $q(y, t-x)$ under the integral sign can be expressed by Equation (7).

Likewise for any point $(x, t)$ in the region $\left\{(x, t) ; t-2 a \leq x \leq t-a, t \geq 0,0 \leq x<a_{m}\right\}$, its solution can be expressed as

$$
\begin{aligned}
& q(x, t)=q(0, t-x) \\
& =\int_{a}^{a_{m}} B(y) q(y, t-x) d y \\
& =\int_{a}^{a_{m}} B(y) d y \int_{a}^{a_{m}} B(s) q_{0}(s-t+x+y) d s .
\end{aligned}
$$

Repeat the above approach, and we can solve it in the whole area $\left\{(x, t) ; 0 \leq x<a_{m}, t \geq 0\right\}$. The following recursion formula can be used:

$$
q(x, t)=\left\{\begin{array}{l}
q_{0}(x-t), x \geq t, \\
\int_{a}^{a_{m}} B(y) q(y, t-x) d y, x<t ;
\end{array}\right.
$$

Then the solution of question (3) is $p(x, t)=e^{-\int_{0}^{x} \mu(\tau) d \tau} q(x, t)$.

Although the solution is obtained in divided area, the solution in the entire region is smooth and satisfies the equation due to the smooth of $q_{0}(x)$ and $B(x)$. 
According to the theory of partial differential equations, in the population development Equation (2), when $\mu(x, t)$, $p_{0}(x), \varphi(t)$ is given, we can get the solution of the population density function $p(x, t)$, by which we can gradually predict the number of population and age structure.

\section{The Optimal Control of Population}

If we complicate the Equation (3), we can set $P(t)$ as the total population at $t ; \Phi(P(t))$ is the external mortality rate, such as earthquake, tidal wave, traffic accident etc., that doesn't rely on age; $u(t)$ is the gaining factor of human population. In this way, we can get the equation

$$
\left\{\begin{aligned}
\frac{\partial p(x, t)}{\partial x} & +\frac{\partial p(x, t)}{\partial t}+\mu(x) p(x, t)+\Phi(P(t)) p(x, t)=-u(t) p(x, t), 0 \leq x<a_{m}, t \geq 0 \\
p(0, t) & =\int_{0}^{a_{m}} b(x) p(x, t) d x, t \geq 0 \\
p(x, 0) & =p_{0}(x), 0 \leq x<a_{m} \\
P(t) & =\int_{0}^{a_{m}} p(x, t) d x, t \geq 0
\end{aligned}\right.
$$

Here, in order to get the solution of (8), we might as well separate the variables:

$$
p(x, t)=y(t) r(x, t),
$$

Thus we can change Equation (8) into (Specific derive are detailed documentation (Li \& Yao, 2006)

$$
\left\{\begin{array}{l}
r_{t}+r_{x}+\mu(x) r=0,0<x<a_{m}, t \geq 0 \\
r(0, t)=\int_{0}^{a_{m}} b(x) p(x, t) d x, t \geq 0 \\
r(x, 0)=p_{0}(x), 0<a<a_{m}
\end{array}\right.
$$

And

$$
\left\{\begin{array}{l}
y^{\prime}(t)+\Phi\left(P_{0}(t) y(t)\right)+u(t) y(t)=0, t \geq 0 \\
y(0)=1
\end{array}\right.
$$

In which $P_{0}(t)=\int_{0}^{a_{m}} r(x, t) d x, t \geq 0$.

Apparently, there is only one solution to the Equations (9) and (10) as it has been proved that the Equation (9) has a unique solution and Equation (10) is an ordinary differential equation which is easy to know it has a unique solution. Therefore, the Equation (8) has a unique solution.

Next, we move to the question of control:

$$
\text { Maximize } J(u):=\int_{0}^{T} m(t) u(t) y^{u}(t)
$$

in which $y^{u}(t)$ is the solution of (10); Here $u(t) \in\{u(t): 0 \leq u(t) \leq v(t), \forall t \in(0, T)\}$, with fixed $v(t) \in L^{1}(0, T)$; $T$ is the upper limit of the time $t ; m(t)=\int_{0}^{a_{m}} g(x) r(x, t) d x, t \in[0, T]$, in which $g(x)$ is a factor that only depends on age and satisfies $g(x) \in L^{\infty}\left(0, a_{m}\right), g(x)>0$.

The main result drawn from this section is:

Theorem (11)-(10) exists an optimal control at least.

Proof. We consider $d=\sup _{u \in A} \int_{0}^{T} m(t) u(t) y^{u}(t) d t$, obviously, $d \in[0,+\infty]$. Make $u_{n} \in A$, so that:

$$
d-\frac{1}{n}<\int_{0}^{T} m(t) u_{n}(t) y^{u_{n}(t)} d t \leq d
$$

This is decided by the definition of the supremum.

Now we will explain weak compactness of $u_{n}$. 
(1) Obviously, in $L^{1}(0, T), A$ has uniformly boundness;

(2) For $\forall \varepsilon>0$, necessarily $\exists \delta>0$, so that when $E \subset(0, T)$ satisfies $\mu(E)<\delta$, then we have: $\int_{E} u_{n}(x) d x<$ $\int_{E} v(x) d x<\varepsilon$, for $\forall u_{n} \in A$;

(3) For $\forall \varepsilon>0$, necessarily $\exists$ compact $k \subset(0, T)$, so that: $\int_{(0, T)-k} u_{n}(x) d x<\int_{(0, t)-k} v(x) d x<\varepsilon$, for $\forall u_{n} \in A$.

Therefore, according to the Douford-Pettis theorm:

(one) $u_{n}$ is at the weak convergence $u^{*}$ in $L^{1}(0, T)$.

We can achieve that:

$$
y^{u_{n}} \rightarrow y^{u^{*}} \text { in } L^{1}(0, T)
$$

Consequently,

$$
m y^{u_{n}} \rightarrow m y^{u^{*}} \text { in } L^{1}(0, T)
$$

With the condition that $m \in L^{\infty}(0, T)$, we can achieve:

(two)

$$
\int_{0}^{T} m(t) u_{n}(t) y^{u_{n}(t)} d t \rightarrow \int_{0}^{T} m(t) u^{*}(t) y^{u^{*}(t)} d t
$$

Combining (one) and (two), we can immediately achieve this:

$$
d=\int_{0}^{T} m(t) u^{*}(t) y^{u^{*}(t)} d t
$$

Thus the $\left(u^{*}, y^{u^{*}}\right)$ is the superior pair of (11)-(10).

Therefore, the original issue has been proved.

\section{Summary}

To sum up, I do some explanations to the optimal control of the stationery population in this paper. I made detailed analysis and obtained the existence of the optimal control for the gain factor of more general case especially. It is feasible and the conclusion is objective.

But the practical value to the conclusion is not high, specific performance in: First of all, in the real world population is generally not stable, and those parameters are generally not having the above properties, this paper is about the demographic idealized vision; Secondly, this paper simply explains the existence of optimal controls, without pointing out how to control them; Finally, the nature of some parameters as well as the characteristic of the gain factor is special in this paper.

Due to lack of funds and other reasons, I will be find a way to try to resolve the problem later.

\section{References}

Anita, S. (1998). Optimal harvesting for a nonlinear age-dependent population dynamics. J. Math. Anal. App., 226, 6-22. http://dx.doi.org/10.1006/jmaa.1998.6064

Anita, S. (2000). Analysis and control of age-dependent population dynamics. Derdrecht: Kluwer Academic Publishers.

Fang, Y., \& Xu, Z. C. (2007). Equations of mathematical physics and special functions. Beijing: Science Press.

Li, M., \& Yao, Y. F. (2006). Nonlinear age-dependent population dynamics. Fuyang Normal College Journal, 4(23), 61-64.

Li, Y. J. (2011). Functional analysis. Beijing: Sciences Press.

Wu, F. T. (2001). Equations of mathematical physics. Wuhan: Wuhan University Press. 